\title{
Exame de Ultrassonografia entre 11 e 14 semanas e Síndrome de Edwards: relato de caso.
}

\section{Ultrasound examination between 11 and 14 weeks and Edwards Syndrome: case report.}

Guilherme de Almeida Bastos ${ }^{1}$

Marcelo Ayres Tambasco ${ }^{3}$

Victor Hugo Benalia ${ }^{3}$

Lais de Paiva Panaino ${ }^{4}$

Letícia Mostaro Pimentel Conde Mostaro ${ }^{5}$

\begin{abstract}
Resumo
Os autores relatam um caso de cromossomopatia, trimossomia do 18, Síndrome de Edwards que foi suspeitado através do exame de ultrassonografia realizado entre 11 e 14 semanas de gestaçấo, em duas oportunidades, que mostrou aumento da transluscência nucal acima de $2,5 \mathrm{~mm}$, a qual foi complementada com a medida do osso nasal e Doppler do ducto venoso e válvula tricuspede, estando todos exames alterados. Diante dessa suspeita diagnóstica, indicou-se um método invasivo para idade gestacional acima de 14 semanas, a amniocentese. No exame laboratorial do líquido amniótico, para pesquisa do cariótipo fetal foi entáo diagnosticado a trissomia do cariotipo: 47,XX,+18, Síndrome de Edwards.
\end{abstract}

Pavavras-chave: Síndrome de Edwards / Aneuploidia / Transluscência Nucal.

\begin{abstract} sed with trisomy karyotype: 47, XX, +18 , Edwards Syndrome.

Keywords: Edwards Syndrome / Aneuploidy / Nuchal Translucency.
\end{abstract}

The authors report a case of chromosoma, trisomy of 18, Edwards Syndrome was suspected by ultrasound scan performed between 11 and 14 weeks of gestation on two occasions, which showed increased nuchal translucency above 2.5 mm, which was complemented with the measurement of nasal bone and ductus venosus Doppler and tricuspid valve, with all abnormal tests. Given this suspected diagnoses was indicated an invasive method for gestational age above 14 weeks, amniocentesis. In the laboratory examination of amniotic fluid for researching fetal karyotype was then diagno-

\footnotetext{
1. Professor Assistente da Disciplina de Obstetrícia do Centro Universitário de Volta Redonda (UNIFOA), aluno do Programa de Mestrado Profissional em Ensino de Ciências da Saúde e do Meio Ambiente. Centro Universitário de Volta Redonda, Conselho de Ensino, Pesquisa e Extensão.

2. Estudante de Medicina do Centro Universitário de Volta Redonda, Conselho de Ensino, Pesquisa e Extensão.

3. Estudante de Medicina do Centro Universitário de Volta Redonda, Conselho de Ensino, Pesquisa e Extensão.

4. Estudante de Medicina do Centro Universitário de Volta Redonda, Conselho de Ensino, Pesquisa e Extensão.

5. Estudante de Medicina do Centro Universitário de Volta Redonda, Conselho de Ensino, Pesquisa e Extensão.
} 


\section{INTRODUÇÃO}

Paciente S.C.S., multípara, 36 anos, oriunda do pré natal de baixo risco, foi matriculada no pré natal de alto risco ao suspeitar-se de uma cromossomopatia. Uma ultrassonografia transvaginal nível I, realizada ao oitavo dia do mês de setembro de 2009, evidenciou um aumento na transluscência nucal igual a 7,9 mm (normal menor que 2,5 mm), CCN de $45 \mathrm{~mm}$, compatível com idade gestacional de 11 semanas. Já no pré-natal de alto risco foi solicitado o exame de ultrassonografia morfológico do primeiro trimestre, nível II, que evidenciou ducto venoso com onda reversa, regurgitação tricuspede e osso nasal normal, medindo 2,6 $\mathrm{mm}$ (normal acima de 1,7 mm, em qualquer idade gestacional). A paciente foi encaminhada para um hospital de referência no Rio de Janeiro, o Instituto Fernandes Figueira, onde foi realizado, um procedimento invasivo, amniocentese, punção do líquido amniótico, após a décima quarta semana de gestação, em que foram analisados 15 metáfases todas com 47 cromososmos pela técnica de bandeamento GTG. Em excesso, um cromossomo do par 18 e diagnóstico de Síndrome de Edwards.

\section{MATERIAS E MÉTODOS:}

A ultrassonografia nível I é um método diagnóstico realizado durante a gestação para rastreamento da idade gestacional, corionicidade e patologias estruturais fetais sendo universalmente utilizada durante toda a gravidez, sem quaisquer problemas para o binômio materno fetal. No primeiro trimestre, existe uma janela acústica entre 11 e 14 semanas com método ultrassonográfico, onde se faz o rastreamento de aneuploidias. Quando se encontra uma translucência nucal igual ou maior que $2,5 \mathrm{~mm}$, solicita-se a ultrassonografia morfológica do $1^{\circ}$ trimestre, nivel II, que consiste na medida do osso nasal, Dopplerfluxometria da válvula tricúspede, ducto venoso e artéria umbilical. Segundo Mauad ( 2006) "O rastreio ecográfico nas semanas 11 e 14 em busca de aneuploidias fetais tem experimentado avanços importantes, especialmente a partir de quando a ecografia foi reconhecida como uma técnica inócua capaz de identificar um subgrupo de gestantes e considerá-las de risco". ${ }^{1}$ De acordo com Carvalho ( 2008) "Proposiçâo mais recente correlaciona alteraçôes na Dopplerfluxometria venosa no final do primeiro trimestre à presença de anormalidades cromossomicas.(...), placenta com alteraçóes cromossomicas apresentam defeito na angiogenese e que fetos portadores de trissomias apresentam cardiopatias estruturais."2

Quando existe uma medida da transluscência nucal, TN maior ou igual a $2,5 \mathrm{~mm}$ a suspeita de cromossomo- patia é de $70 \%$ que se eleva para $90 \%$ se tiver agnesia o hipoplasia do osso nasal e se o Dopplerfluxometria da válvula tricúspide e ducto venoso e artéria umbilical se encontrarem reversos, é sugestivo de cardiopatias que acompanham em $50 \%$ dos casos dos fetos com trissomias sendo entáo necessário uma metodologia invasiva de 10 a 12 semanas como a biópsia da vilo corial, acima de 14 semanas até 18 semanas amniocentese e depois desta data gestacional a cordocentese. Segundo Freitas (2006) "A medida da TN alterada constitui uma suspeita diagnóstica de aneuploidias em $70 \%$ dos casos e quando não existe trissomia fetal ao exame do cariótipo do liquido amniótico, geralmente o feto cursa com outras patologias estruturais fetais, por isso é indispensável a realização de métodos invasivos conforme a idade gestacional para elucidação diagnóstica de patologias fetais.

No segundo trimestre, o exame de ultrassonografia, nível I, deve ser realizado entre 20 e 24 semanas para rastreamento de patologias estruturais fetais, e em casos suspeitos a gestante deve ser encaminhada para ultrassonografia nível II, ultrassonografia morfológica do $2^{\circ}$ trimestre a qual permite um estudo detalhado das doenças suspeitadas. No terceiro trimestre, entre 30 e 34 semanas, a ultrassonografia é útil para evidenciar patologias placentárias, maturidade precoce quando se encontra com grau II e III de GRANUM antes de 32 e 35 semanas, respectivamente. Do desenvolvimento fetal com percentil diminuído constituindo os fetos pequenos para idade gestacional (PIG), CIUR simétrico ou assimétrico e grande para a idade gestacional (GIG) acima do percentil 90. Como também para o acompanhamento dos fetos adequados para a idade gestacional (AID), entre 10 e 90 do percentil. Nos casos patológicos o acompanhamento deverá ser feito cardiotocografia basal anteparto e / ou Dopplerfluxometria para avaliação da vitalidade fetal. O estudo fetal realizado durante toda a gravidez através do exame de ultrassonografia como método diagnóstico e até de tratamento fetal, constitui uma nova especialidade médica, a Medicina Fetal.

\section{DISCUSSÁO E RESULTADOS}

Paciente S.C.S., 36 anos, multípara, G-VIII, P-IV, AIII, ao suspeitar de uma gravidez procurou o Sistema Único de Saúde (SUS) em Volta Redonda e encaminhada ao pré-natal de baixo risco. Após o resultado dos exames de primeira rotina e ultrassonografia alterada, foi transferida para o pré-natal de alto risco. A ultrassonografia realizada em 02/09/2009, foi compatível com 11 semanas e 2 dias de gestação revelando um padrão aumentado de trasnluscência nucal de 3,6 mm. Aos cuidados do pré-natal de alto risco foi solicitada uma nova ultrassonografia em 08/09/2009, 
que confirmou o aumento da transluscência nucal em 7,9 mm. Com os dados encontrados a gestante foi encaminhada para o centro de referência, Instituto Fernandes Figueira para a realização de método invasivo, amniocentese e pesquisa de cariótipo. O cariótipo obtido do líquido amniótico, foi analisado e revelou 15 metáfases, todas com 47 cromososmos (não seria cromossomos?) pela técnica de bandeamento GTG. Em excesso, um cromossomo do par 18, confirmando a existência de uma trissomia do cromossomo 18 , cujo cariótipo encontrado foi $47, \mathrm{XX},+18$, Síndrome de Edwards.

Após a confirmação da cromossomopatia, o pré natal de alto risco foi seguido até o dia do parto. A gestação cursou com crescimento intra uterino restrito (CIUR) e no ecocardiograma fetal realizado em 19/03/2010, com idade gestacional avançada de 37 semanas e 2 dias, quando deveria ter sido realizado entre 26 e 30 semanas de gestação, evidenciou um septo interventricular com pequena comunicação e valva aórtica displásica. Ao vigésimo sexto dia de março de 2010, gestante em parto normal deu a luz um feto vivo, único, do sexo feminino, em péssimas condiçôes de vida, infectado com APGAR 4/7, capurro somático 38 semanas e 2 dias, assistido, aspirado, entubado e encaminhado a UTI Neo-Natal.

Ao exame detalhado o recém-nascido era pequeno para idade gestacional, $1755 \mathrm{~g}$, fascies, olho, pupila e pescoço anormais e aparelho respiratório com ritmo irregular, ausculta normal e dificuldade respiratória leve. $\mathrm{O}$ recém-nascido ficou bem ao nascer, e ao terceiro dia de vida evoluiu com cianose progressiva e queda da pressão arterial. Foi solicitado um ecocardiograma que revelou persistência do canal arterial, comunicação interventricular e miocardiopatia dilatada. O recém-nascido permaneceu na UTI até seu quarto dia de vida, vindo ao óbito às $10 \mathrm{~h} 40 \mathrm{~min}$. do dia 30/03/2010, devido a uma parada cardiorespiratória em decorrêwncia de suas malformaçóes cardíacas.

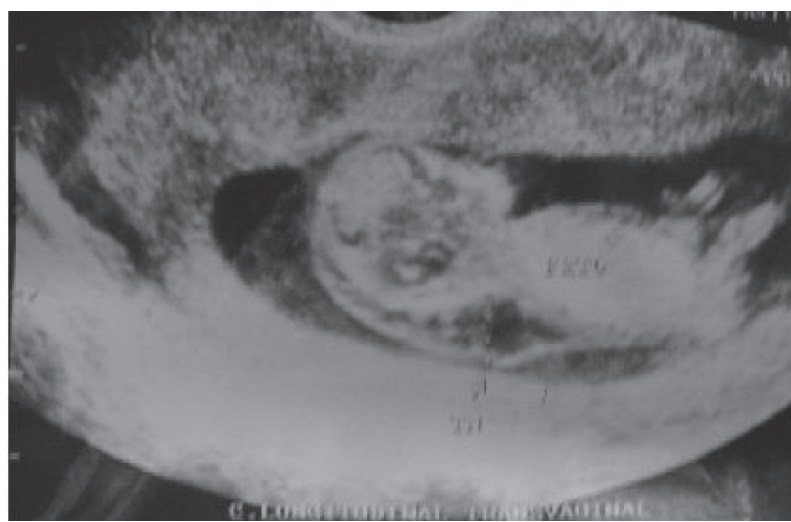

Figura1 - Ultrassonografia realizada entre 11 e 14 semanas de gestaçâo. Valores de C.S.N. $=45 \mathrm{~mm}$ e T.N. $=7,9 \mathrm{~mm}$.

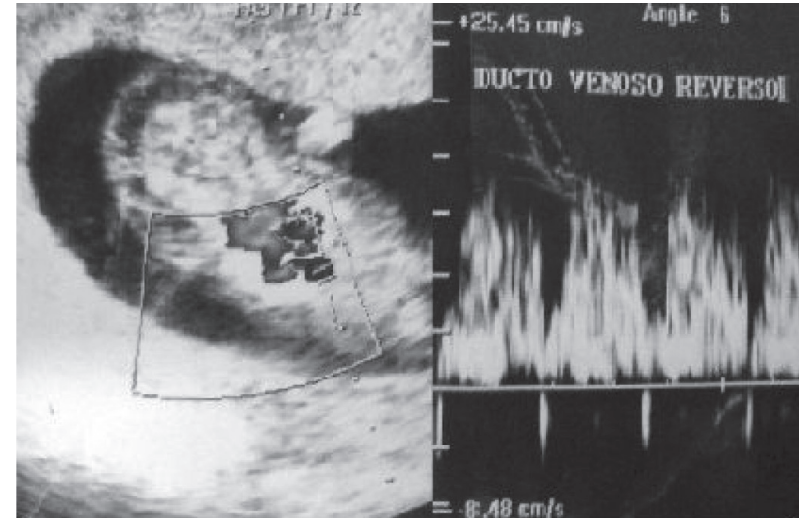

Figura2 - Ducto Venoso Reverso

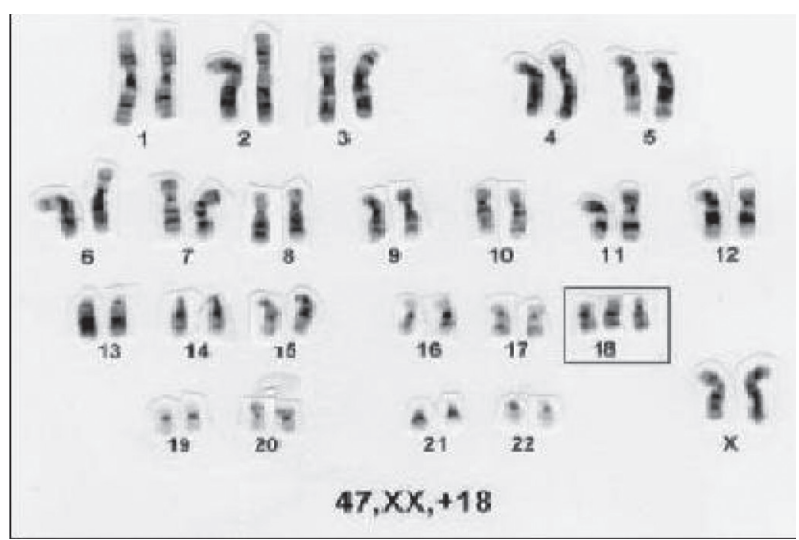

Figura3 - Cariótipo de 47, XX, +18, sendo compatível Com Sindrome de Edwards.

\section{CONCLUSÃO}

A ultrassonografia obstétrica, nível I, é um exame essencial para acompanhamento no pré-natal das gestantes de baixo risco com a finalidade de identificar patologias diagnósticas, gemelaridade e corrigir idade gestacional, identificando, desta forma, grupo de risco e transferindo-as para o pré-natal de alto risco, onde elas serão submetidas a exames de ultrassonografia obstétrica nível II. No primeiro trimestre de gestação para o rastreamento de cromossomopatias, a ultrassonografia obstétrica deverá sempre ser realizada na janela acústica de 11 a 14 semanas e comprimento cabeça nádega fetal $(\mathrm{CCN})$ de 45 a $84 \mathrm{~mm}$, identificando uma translucência nucal normal menor que $2,5 \mathrm{~mm}$. Valores iguais ou acima de $2,5 \mathrm{~mm}$ são considerados alterados e indicado complementaçáo do exame ultrassonográfico com medida do osso nasal, Dopplerfluxometria da artéria umbilical, do ducto venoso e válvula tricúspide, o chamado exame obstétrico morfológico do primeiro trimestre. Caso esse exame morfológico se encontre com seus fluxos alterados, deve-se proceder a realização de método invasivo, biópsia do vilo corial, amniocentese ou cordocentese, dependendo da idade gestacional, para esclarecer a presença ou não de trissomia e caracterizando-a, se do $21,18,13$, etc. 
No caso relatado, houve o cumprimento de toda essa sequência diagnóstica e identificação do cariótipo fetal pela análise do liquido amniótico, identificando-a como do sexo feminino e portadora de trissomia do cromossomo 18, Síndrome de Edwards. Embora tardiamente, foi realizado no pré-natal de alto risco a ecocardiografia fetal demonstrando cardiopatia congênita. Foram feitos outros exames de ultrassonografias obstétricas no segundo e terceiro trimestre da gestaçáo, evidenciando um percentil de crescimento abaixo de 10, portanto CIUR simétrico. Por ser uma multípara, a mesma deu a luz de parto normal a termo, capurro de 38 semanas e 4dias, recém nascido $(\mathrm{RN})$ de baixo peso e portador de cardiopatia congênita grave, vindo a falecer alguns dias após o parto. Isso demonstra a importância do exame de ultrassonografia realizado na gestação e o horizonte de procedimentos em beneficio da mãe e do feto, podendo se programar as abordagens e orientar os famliares sobre outros recursos que deveráo ser utilizados no pós parto imediato. Neste caso, houve a suspeita, a confirmação diagnóstica e o parto normal apesar da patologia fetal, Síndrome de Edwards.

\section{REFERÊNCIAS BIBLIOGRÁFICAS}

1. AGUIAR, R.A.L.P. ; PENA, S.J. . Tratado de Obstetrícia FEBRASGO: Diagnóstico pré natal, A Visão do Geneticista. Rio de Janeiro - RJ: Livraria e Editora Revinter Ltda., 2001, Cap.: 80.
2. BENACERRAF, B. R. Diagnósticos Ultra-Sonográfico das Sindromes Fetais. Trad. sob direção de José Eduardo Ferreira de Figueiredo. Rio de Janeiro - RJ: Revinter, 2001. 466 p.

3. BRIZOT, N.L. ;CARVALHO, M.H.B. ;LIAO, A.W. . Zugaib Obstetricia: Medicina Fetal. 1. Ed. Barueri SP: Editora Manole Ltda., 2008, Cap.:7

4. FREITAS, F. ; Rotinas em Obstetrícia: Ultrassonografia Obstétrica. 5. ed. Porto Alegre - RS: Editora Artmed, 2006.

5. MAUAD FILHO, F. ; PINHEIRO FILHO, L. S. . Ultra-Sonografia na Prática Obstétrica. 1. ed. São Paulo SP: Livraria e Editora Revinter Ltda., 2006. v. 1. 396 p.

6. NICOLAIDES, K. H.; SEBIRE, N. J.; SNIJDERS, R. J. M. O exame ultra-sonográfico entre 11-14 semanas: Diagnósticos de anomalias fetais. Trad. sob direção de Maria de Lourdes Brizot. Cosenza (Italy): Parthenon Publishing Group, 1999. 194 p.

7. NICOLAIDES, K. H.; SEBIRE, N. J.; SNIJDERS, R. J. M. O exame ultra-sonográfico entre 18-23 semanas: Diagnósticos de anomalias fetais. Trad. sob direção de Maria de Lourdes Brizot. Cosenza (Italy): Parthenon Publishing Group, 1999. 126 p. 